\title{
Investigation of Adverse Cancer Related DNA Methylation Variation in the Human Telomerase Reverse Transcriptase (hTERT) Promotor in Follicular Fluid Cells of Women Subjected to Ovarian Stimulation for In Vitro Fertilization (IVF)
}

\author{
Comhaire $\mathrm{FH}^{1 *}$, Decleer WAE ${ }^{2}$, Declerck $\mathrm{K}^{3}$ and Vanden Berghe $\mathbf{W}^{3}$ \\ ${ }^{1}$ Fertility-Belgium Clinic, Weststraat, 16, 9880 Aalter, Belgium \\ ${ }^{2}$ Centre for Fertility, AZ Jan Palfijn Gent, Watersportlaan 5, 9000 Gent, Belgium \\ ${ }^{3}$ Lab Protein Science, Proteomics and Epigenetic Signaling, Department of Biomedical Sciences, University Antwerp, Campus Drie Eiken, Universiteitsplein 1, Wilrijk, \\ 2610, Belgium
}

\begin{abstract}
Purpose: To determine the variability in DNA methylation of the human telomerase reverse transcriptase (hTERT) promotor in cells of follicular fluid of patients receiving ovarian stimulation for treatment with in vitro fertilization (IVF), in connection with possible cancer related health risk in offspring.
\end{abstract}

Materials and method: The pilot study analysed the variability in DNA methylation of five cancer related CpG biomarkers in the hTERT promoter by bisulfite pyrosequencing in cells of follicular fluid of 8 patients treated by IVF, each one originating from one ovary.

Results: The absolute promoter methylation levels of individual CpG dinucleotides in the hTERT promoter region in cells of follicular fluid of patients undergoing ovarian stimulation for IVF varied between 2 and $15 \%$. Although the mean hTERT promoter methylation did not significantly differ from levels observed in healthy control samples, maximal DNA methylation levels of one tumour specific $\mathrm{CpC}$ methylation biomarker were close to levels detected in hepatocellular cancer and in paediatric brain tumours.

Conclusion: Bisulfite pyrosequencing allows for highly sensitive detection of CpG specific DNA methylation of the five dinucleotides of the hTERT promoter region in cells of follicular fluid of patients treated by ovarian stimulation for IVF. The occasional observation of tumorigenic DNA methylation at GpC motifs strongly associated with hepatocellular carcinoma and sporadic malignant paediatric brain tumours, suggests that offspring of IVF pregnancies may be at increased risk of adverse cancer outcomes. In order to reduce the possible cancer risk in the offspring related to hTERT hypermethylation and oxidative DNA damage, nutraceutical food supplementation during conception and early pregnancy may warrant further investigation, aiming at the reduction of the homocysteine concentration and the hTERT promoter methylation in follicular fluid and cells during ovarian stimulation.

Keywords: hTERT-promotor; DNA methylation; Follicular fluid; Nutraceutical food supplement; In vitro fertilization; Epigenetics

\section{Introduction}

Assisted reproduction techniques, in particular IVF and ICSI, have generated enormous progress to the treatment of couple infertility. However, some doubts have risen regarding the health and development of children born after using these techniques. This involves aspects of transition mutagenesis and potential carcinogenesis related to the excess of oxidized DNA (8-OH-2deoxyguanosin) in spermatozoa of infertile men [1], and metagenetic alterations possibly causing unfavourable cardio-metabolic profiles in childhood [2], and increased risk for drug treated attention deficit/hyperactivity disorder [3].

Ovarian stimulation for IVF has been reported to cause increased concentrations of homocysteine in blood and in follicular fluid [4,5] that, among other things, is associated with poor embryo quality [6,7] and with unfavourable pregnancy outcomes. Elevated homocysteine may influence DNA methylation [8,9], particularly at the CpG sites [10]. It should be stressed that homocysteine may either decrease or increase DNA methylation through the one carbon pathway. Aside from the effect of oestrogens, external factors, nutrition in particular [11], influence the balance between the two main cofactors S-adenosylmethionine (SAM) and S- adenosylhomocysteine (SAH) in transferring methyl groups to the DNA [9].

In mature human oocytes telomerase remains quiescent [12] and telomerase activity is low or absent $[13,14]$, but telomerase reverse transferase (TERT)-mRNA is expressed in human oocytes at the stage of germinal vesicle, metaphase I, metaphase II during the cycle of ovarian stimulation, and in cleavage-stage embryos. In addition, three different TERT subunit isoforms were found in oocytes [15]. Telomeres are shorter in the oocytes from women undergoing in vitro fertilization, who then produced fragmented, aneuploidy embryos that fail to implant [16], and higher telomerase activity was a more significant predictor of a favourable outcome of IVF treatment than telomere length in granulosa cells [17].

Recent reports suggest the prevalence of certain cancers and of leukaemia to be increased among children born after IVF [18-20].

*Corresponding author: Frank H Comhaire, Brakelmeersstraat, 18, B 9830 Sint Martens-Latem, Belgium, Tel: 0032475618 555; E-mail: frank@comhaire.com

Received September 28, 2015; Accepted October 27, 2015; Published November 03, 2015

Citation: Comhaire FH, Decleer WAE, Declerck K, Vanden Berghe W (2015) Investigation of Adverse Cancer Related DNA Methylation Variation in the Human Telomerase Reverse Transcriptase (hTERT) Promotor in Follicular Fluid Cells of Women Subjected to Ovarian Stimulation for In Vitro Fertilization (IVF). JFIV Reprod Med Genet 3: 161. doi:10.4172/2375-4508.1000161

Copyright: (C) 2015 Comhaire $\mathrm{FH}$, et al. This is an open-access article distributed under the terms of the Creative Commons Attribution License, which permits unrestricted use, distribution, and reproduction in any medium, provided the original author and source are credited. 
Citation: Comhaire FH, Decleer WAE, Declerck K, Vanden Berghe W (2015) Investigation of Adverse Cancer Related DNA Methylation Variation in the Human Telomerase Reverse Transcriptase (hTERT) Promotor in Follicular Fluid Cells of Women Subjected to Ovarian Stimulation for In Vitro Fertilization (IVF). JFIV Reprod Med Genet 3: 161. doi:10.4172/2375-4508.1000161

There is evidence that childhood brain cancers are characterised by increased methylation of the promotor region of the telomerase reverse transcriptase (Figure 1) increasing telomerase enzymatic activity [21]. The TERT-promoter regulates the activity of the catabolic subunit of telomerase. Hyper-methylation of the TERT promoter region results in decreased activity of this catabolic subunit [22], by which the anabolic subunit will become predominant [23]. This may result in excessive lengthening of the telomeres, which is associated with malignant degeneration [24].

It is our hypothesis that altered methylation of the TERT promotor region, possibly related to increased homocysteine concentration in follicular fluid of stimulated ovaries in IVF, may be a factor associated to the observed increment in cancers in the offspring (Figure 2). In order to test the hypothesis a study was performed, to develop and validate a pyrosequencing method to quantify the DNA methylation levels of 5 cancer related CPG biomarkers in the hTERT promoter region in follicular fluid obtained during oocyte retrieval in IVF.

Of particular interest in relation to protecting the offspring of IVF against undesired (epi-)genetic defects, nutraceutical food supplementation to the father was found to decrease the sperm content of oxidised DNA [1], whereas the preconception and gestational supplementation to the mother increased the ongoing pregnancy rate after IVF [25]. Also, early gestation is considered the critical timewindow for changes in the prenatal environment and to affect the adult human blood methylation [26]. Nutraceutical food supplements for the treatment of couple infertility to be taken around conception have recently been compared [27]. Moreover, we have been the first to introduce a formulation containing antioxidants, plant extracts and the combination of vitamins $\mathrm{B} 6, \mathrm{~B} 9$ and $\mathrm{B} 12$, reducing the homocysteine concentration, and selenium [28]. The latter has been

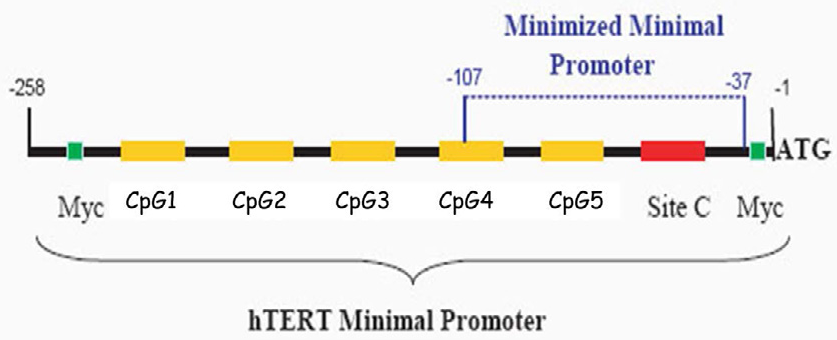

Figure 1: Schematic representation of $\mathrm{CpG}$ motif positions relative to the hTERT promoter (UTSS region upstream of the transcription start site), selected for $\mathrm{CpG}$ pyrosequencing analysis.

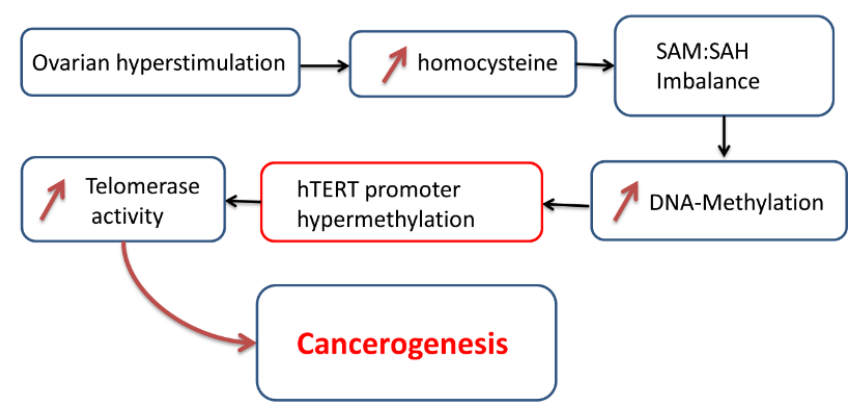

Figure 2: Flow chart representing the hypothesis whereby ovarian stimulation may induce increased methylation of the hTERT promoter region, causing excessive lengthening of telomeres, possibly increasing the risk of malignant pathology among IVF offspring. SAM: S-adenosylmethionine, SAH: S-adenosylhomocysteine. shown to decrease DNA-methylation [29-31] by inhibiting the activity of the methyltransferase [32]. The hTERT promoter upstream of the transcription start site (UTSS region) consists of 5 well characterized CpG dinucleotides (Figure 1).

\section{Materials and Method}

Eight samples of follicular fluid were obtained at the occasion of the oocyte retrieval for IVF. Patients were stimulated to develop several follicles according to conventional methods. The patients were treated for ovarian stimulation with human menopausal gonadotropin (hMG, Menopur ${ }^{\circledR}$, Ferring Pharmaceuticals, Saint Prex, Switzerland), three ampules of $75 \mathrm{IU}$ subcutaneously daily until a follicular diameter of at least $18 \mathrm{~mm}$ was attained. Triggering oocyte maturation was done with 10000 IU of human chorionic gonadotropin (hCG, Pregnyl, MSD, Kenilworth, New Jersey, United Sates) injected 36 hours before oocyte pick-up. Aspiration of the follicular fluid was performed through a Cook 17 gauge aspiration needle (K-OSN-1730-B-60) with a negative pressure of $140 \mathrm{~mm}$ HG (Cook vacuum pump UL 60601-1, CAN/CSA-C22.2). The cumulus oocyte complexes were isolated and transferred into a culture medium (Ferticult IVF medium ${ }^{\circledR}$, Fertipro, Beernem, Belgium). The remaining follicular fluid - on the condition that it was macroscopically free of blood - of the different follicles of a single ovary were added in one container per individual patient. These samples were immediately pelleted by centrifuged $(3000 \mathrm{rpm} 10 \mathrm{~min})$ and transported snap-frozen on dry ice and stored at $-70^{\circ} \mathrm{C}$. Genomic DNA was subsequently isolated from frozen pellets with DNeasy blood and tissue kit (Qiagen).

Epigenetic analysis for the measurement of the degree of methylation of the hTERT promotor and its 5 individual dinucleotides was performed essentially as described by Castelo-Branco et al. [21] with minor modifications. In brief, $1 \mu \mathrm{g}$ gDNA of each sample was sodium bisulfite converted using the EpiTect fast DNA bisulfite kit (Qiagen) according to manufacturer's instructions. $15 \mathrm{ng}$ bisulfite converted DNA was used as an input for PCR amplification using the PyroMark PCR kit (Qiagen). Finally, pyrosequencing was performed using the PyroMark Q24 instrument and PyroMark Q24 advanced CpG reagents (Qiagen). Methylation percentages for each CpG were obtained and analysed using the PyroMark Q24 advanced software (Qiagen). Only samples which passed quality control after pyrosequencing were used for further analysis and graphical representation. The hTERT pyrosequencing assay was designed using the PyroMark assay design software (Qiagen). Following primers were used: forward ATGTGGAGGTTTTGGGAATAG, reverse AACCTAAAAACAACCCTAAATCT and sequencing GGTTTTGGGAATAGGTG, spanning the hTERT promoter region (chr5:1,295,586-1,295,643).

Simultaneously positive reference gDNA material isolated from highly metastatic MDA-MB231 breast cancer cell lines was analysed for cancer-related DNA-hyper-methylation, to serve as internal validation of the epigenetic test procedure.

Since it seems ethically unacceptable or technically unfeasible to aspirate follicular fluid in healthy women not treated by assisted reproduction, the experimental setup does not include follicular fluid of untreated controls. gDNA depleted samples served as negative test controls and did not yield any signal (data not shown). The results were plotted and analysed by the MedCalc statistical programme (MedCalc, Ostend, Belgium), and presented as multiple line graphs.

The research was approved by the ethical committee of the Palfijn Hospital, Ghent, Belgium, and patients gave informed consent to participating in the project. 
Citation: Comhaire FH, Decleer WAE, Declerck K, Vanden Berghe W (2015) Investigation of Adverse Cancer Related DNA Methylation Variation in the Human Telomerase Reverse Transcriptase (hTERT) Promotor in Follicular Fluid Cells of Women Subjected to Ovarian Stimulation for In Vitro Fertilization (IVF). JFIV Reprod Med Genet 3: 161. doi:10.4172/2375-4508.1000161

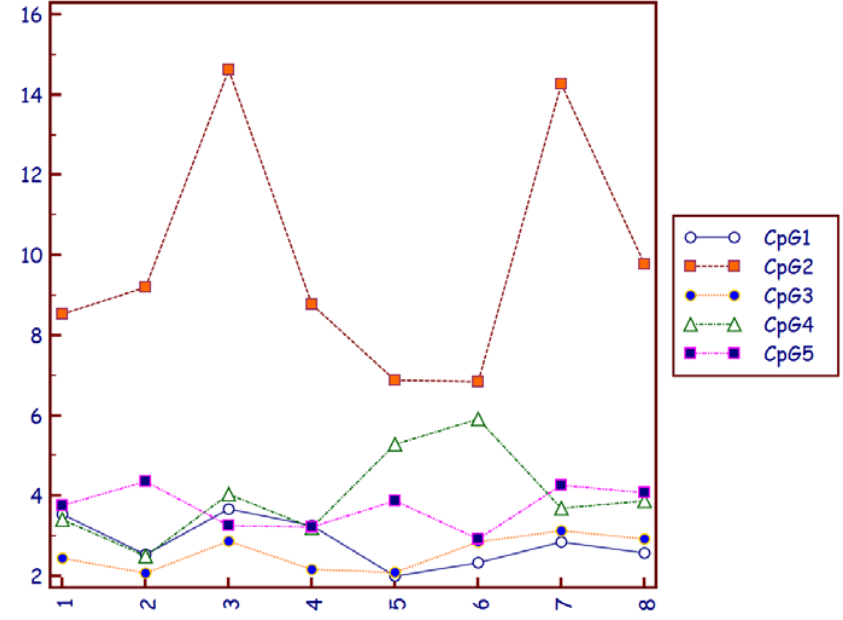

Figure 3: Graph representing the absolute DNA methylation levels of $5 \mathrm{CpG}$ motifs encompassing the hTERT UTSS promoter region (in \% on the vertical axis), as determined by bisulfite pyrosequencing of 8 samples of follicular fluid (indicated by their numbers on the horizontal axis). The results are presented as multiple line diagrams, with lines graphically connecting the degree of methylation of the individual dinucleotides per sample.

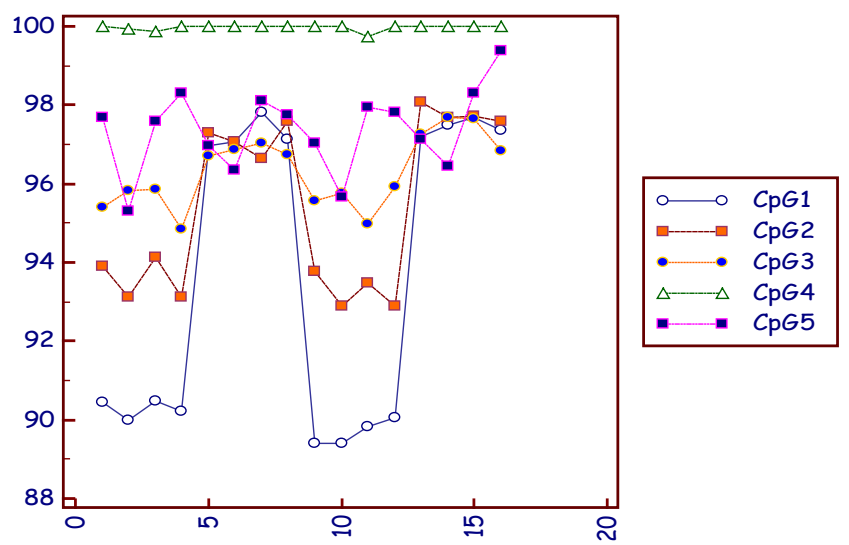

Figure 4: Graph representing the absolute DNA methylation levels of $5 \mathrm{CpG}$ motifs encompassing the hTERT UTSS promoter region (in \% on the vertical axis), as determined by bisulfite pyrosequencing of reference control samples originating from metastatic breast cancer cells. Legend as in Figure 3.

\section{Results}

Figure 3 illustrates absolute CpG specific DNA methylation levels observed in the 5 dinucleotides of gDNA isolated from follicular fluid cells from 8 patients who received ovarian stimulation.

Figure 4 Illustrates DNA methylation values detected in gDNA isolated from highly metastatic MDA-MB231 breast cancer cells (ATCC ${ }^{\oplus} \mathrm{HTB}-26^{\mathrm{TM}}$ ).

It appears that the degree of methylation of the $5 \mathrm{CpG}$ dinucleotides in follicular fluid samples varies between 2 and 15\%, with the highest variability at the CpG2 position (Figure 3). In contrast, DNA methylation levels measured in the metastatic breast cancer cells peaks around 90 to $100 \%$ for all CpGs (Figure 4).

\section{Discussion}

Follicular fluid obtained at the time of puncture of stimulated ovaries during IVF reflects the composition of the "milieu interieur" [33] of metaphase II oocytes and their surrounding corona cells. DNA analysis and measurement of the degree of methylation relates to the nucleated corona radiata and granulosa cells present in follicular fluid. Because of the intense communication between cumulus-corona cells and the oocyte, either paracrine or through gap-junctions [34], the latter is assumed to be representative for the DNA situation of the oocytes. This assumption is justified since the external factors that determine the level of epigenetic changes are common to all cells contained in the follicle, including the oocytes.

The present study is the first to apply $\mathrm{CpG}$ pyrosequencing for the quantification of cancer-related $\mathrm{CpG}$ methylation levels of the hTERT promoter in follicular fluid of patients treated by ovarian stimulation for IVF. Though the DNA methylation in follicular fluid cells is significantly lower than in the control cancerous cells $(\mathrm{P}<0.001$ for each $\mathrm{CpG}$ motif $)$, with an average hTERT promoter DNA methylation of approximately 4.3\% (SD: $0.7 \%$ ), $15 \%$ DNA methylation could be detected for CpG2 in 2 out of 8 samples. The average DNA methylation is in full agreement with DNA methylation results observed in healthy tissue samples, such as haematopoietic stem cells, placenta, lymphocytes, neural stem cells, fibroblasts [21].

With respect to the sporadic 15\% DNA methylation levels observed in the $\mathrm{GpC} 2$ motif, the same $\mathrm{CpG}$ dinucleotide was recently identified as a very reliable cancer biomarker $(\mathrm{P}<0.001)$ for hepatocellular carcinoma, with a mean DNA methylation of $18 \%$ as compared to $7 \%$ in healthy control tissue [24]. Also, 15\% DNA methylation was found to be the limit above which sporadic malignant paediatric brain tumours have been identified, and TERT-promoter methylation was considered relevant. Whereas DNA hyper-methylation over $60 \%$ and increased TERT expression can be observed in most malignant tumours [21], occasional increase $(>15 \%)$ in DNA methylation levels at the CpG2 position in the hTERT promoter in cells present in follicular fluid of stimulated ovaries of IVF patients, may be connected to an increased risk of adverse cancer outcomes in offspring.

As with the variable concentration of homocysteine in follicular fluid [35] there is considerable variability of the degree of DNA methylation of the TERT promotor region in samples of follicular fluid originating from different ovaries. Further study of the relation between homocysteine and hTERT promoter methylation in follicular fluid of stimulated ovaries is mandatory, since the authors are fully aware that the preliminary findings reported here need to be further explored. Nonetheless, it should be prudent to aim at reducing the level of homocysteine and of the degree of DNA-methylation, for instance by pre-conceptional nutraceutical food supplementation to women treated by IVF. Whether prescription of a nutraceutical containing a judicious combination of a panel of anti-oxidants with complementary effects, of plant extracts with anti-inflammatory action, of vitamins B6, B9 and B12 to reduce the homocysteine concentration [27], and of selenium to diminish the level of DNA methylation in couples being treated by IVF, remains to be explored.

\section{Conclusion}

We provide proof of concept for bisulfite pyrosequencing based quantification of $\mathrm{CpG}$ specific DNA methylation in the hTERT promoter of cells of follicular fluid of stimulated ovaries during IVF. In general, average DNA methylation of the hTERT promoter region is relatively low in follicular fluid cells as compared to that in malignant tumour cells, and it is similar to that in healthy cells. In contrast, the degree of methylation at the cancer related CpG2 motif is rather variable, occasionally exceeding the methylation level associated 
Citation: Comhaire FH, Decleer WAE, Declerck K, Vanden Berghe W (2015) Investigation of Adverse Cancer Related DNA Methylation Variation in the Human Telomerase Reverse Transcriptase (hTERT) Promotor in Follicular Fluid Cells of Women Subjected to Ovarian Stimulation for In Vitro Fertilization (IVF). JFIV Reprod Med Genet 3: 161. doi:10.4172/2375-4508.1000161

with hepatocellular carcinoma or sporadic paediatric malignant brain tumours. In this respect strategies aiming at the reduction the concentration of homocysteine, of the levels of DNA methylation, and of oxidative DNA oxidation, both before conception and in early pregnancy deserve further investigation.

\section{Conflict of Interest}

Frank Comhaire holds the Belgian patent protecting the formulation of the nutraceutical referred to in the manuscript.

\section{Acknowledgment}

This study has benefited from financial support of Nutriphyt Ltd, Oostkamp, Belgium. The authors express their gratitude to the technical staff of the FertilityBelgium centre for reproductive medicine of the Jan Palfijn municipal hospital in Ghent.

\section{References}

1. Comhaire $\mathrm{FH}$, Christophe $\mathrm{AB}$, Zalata AA, Dhooge WS, Mahmoud AM, et al (2000) The effects of combined conventional treatment, oral antioxidants and essential fatty acids on sperm biology in subfertile men. Prostaglandins Leukot Essent Fatty Acids 6: 159-165.

2. Painter RC, Grooten IJ, van der Post JA, Mol BW, Vrijkotte TGM, et al. (2015) Subfertility and assisted reproduction techniques are associated with poore cardiometabolic profiles in childhood. Reprod Biomed online 30: 258-267.

3. Källén AJ, Finnström OO, Lindam AP, Nilsson EM, Nygren KG, et al. (2011) Is there an increased risk for drug treated attention deficit/hyperactivity disorder in children born after in vitro fertilization? Eur J Paediatr Neurol 15: 247-253.

4. Boxmeer JC, Steegers-Theunissen RP, Lindemans J, Wildhagen MF, Martin $\mathrm{E}$, et al. (2008) Homocysteine metabolism in the pre-ovulatory follicle during ovarian stimulation. Hum Reprod 23: 2570-2576.

5. Pacchiarotti A, Mohamed MA, Micara G, Linari A, Tranquilli D, et al. (2007) The possible role of hyperhomocysteinemia on IVF outcome. J Assis Reprod Genet 24: $459-462$

6. Berker B, Kaya C, Aytac R, Satiroglu H (2009) Homocysteine concentrations in follicular fluid are associated with poor oocyte and embryo qualities in polycystic ovary syndrome patients undergoing assisted reproduction. Hum Reprod 2: 2293-2302.

7. Ocal P, Ersoylu B, Cepni I, Guralp O, Atakul N, et al. (2012) The association between homocysteine in the follicular fluid with embryo quality and pregnancy rate in assisted reproductive techniques. J Assist Reprod Genet 29: 299-304.

8. Krishna SM, Dear A, Craig JM, Norman PE, Golledge J (2013) The potentia role of homocysteine mediated DNA methylation and associated epigenetic changes in abdominal aortic aneurysm formation. Atherosclerosis 228: 295305

9. Mandaviya PR, Stolk L, Heil SG3 (2014) Homocysteine and DNA methylation: a review of animal and human literature. Mol Genet Metab 113: 243-252.

10. Kinoshita M, Numata S, Tajima A, Shimodera S, Imoto I, et al. (2013) Plasma total homocysteine is associated with DNA methylation in patients with schizophrenia. Epigenetics 8: 584-590.

11. Alshatwi AA, Shafi G (2012) Effect of dietary nutrients on DNA methylation and imprinting. In: Tatarinova T, Kerton O (Eds), DNA Methylation - from Genomics to Technology. InTech, Shangai, China.

12. Keefe DL, Liu L, Marquard K (2007) Telomeres and aging-related meiotic dysfunction in women. Cell Mol Life Sci 64: 139-143.

13. Liu L, Bailey SM, Okuka M, Muñoz P, Li C, et al. (2007) Telomere lengthening early in development. Nat Cell Biol 9: 1436-1441.

14. Ozturk S, Sozen B, Demir N (2014) Telomere length and telomerase activity during oocyte maturation and early embryo development in mammalian species. Mol Hum Reprod 20: 15-30.

15. Brenner CA, Wolny YM, Adler RR, Cohen J (1999) Alternative splicing of the telomerase catalytic subunit in human oocytes and embryos. Mol Hum Reprod 5: $845-850$.

16. Kalmbach KH, Fontes Antunes DM, Dracxler RC, Knier TW, Seth-Smith ML, et al. (2013) Telomeres and human reproduction. Fertil Steril 99: 23-29.

17. Wang W, Chen H, Li R, Ouyang N, Chen J, et al. (2014) Telomerase activity is more significant for predicting the outcome of IVF treatment than telomere length in granulosa cells. Reproduction 147: 649-657.
18. Källén B, Finnström O, Lindam A, Nilsson E, Nygren KG, et al. (2010) Cancer risk in children and young adults conceived by in vitro fertilization. Pediatrics 126: $270-276$

19. Moll AC, Imhof SM, Cruysberg JR, Schouten-van Meeteren AY, Boers M, et al (2003) Incidence of retinoblastoma in children born after in-vitro fertilisation. Lancet 361: 309-310.

20. Petridou ET, Sergentanis TN, Panagopoulou P, Moschovi M, Polychronopoulou $S$, et al. (2012) In vitro fertilization and risk of childhood leukemia in Greece and Sweden. Pediatr Blood Cancer 58: 930-936.

21. Castelo-Branco P, Choufani S, Mack S, Gallagher D, Zhang C, et al. (2013) Methylation of the TERT promoter and risk stratification of childhood brain tumours: an integrative genomic and molecular study. Lancet Oncol 14: 534542.

22. Shin KH, Kang MK, Dicterow E, Park NH (2003) Hypermethylation of the hTERT promoter inhibits the expression of telomerase activity in normal oral fibroblasts and senescent normal oral keratinocytes. $\mathrm{Br} \mathrm{J}$ Cancer 89: 1473-1478.

23. Guilleret I, Yan P, Grange F, Braunschweig R, Bosman FT, et al. (2002) Hypermethylation of the human telomerase catalytic subunit (hTERT) gene correlates with telomerase activity. Int J Cancer 101: 335-341.

24. Zhang H, Weng X, Ye J, He L, Zhou D, et al. (2015) Promoter hypermethylation of TERT is associated with hepatocellular carcinoma in the Han Chinese population. Clin Res Hepatol Gastroenterol 39: 600-609.

25. Comhaire F, Decleer W (2012) Comparing the effectiveness of infertility treatments by numbers needed to treat (NNT). Andrologia 44: 401-404.

26. Tobi EW, Slieker RC, Stein AD, Suchiman HE, Slagboom PE, et al. (2015) Early gestation as the critical time-window for changes in the prenatal environment to affect the adult human blood methylome. Int J Epidemiol 44: 1211-1223.

27. Comhaire FH (2015) Comparison of three nutriceutical food supplements for the treatment of infertility. Br J Phar Res 5: 173-180.

28. Comhaire FH, Mahmoud A (2003) The role of food supplements in the treatment of the infertile man. Reprod Biomed Online 7: 385-391.

29. Davis CD, Uthus EO (2004) DNA methylation, cancer susceptibility, and nutrient interactions. Exp Biol Med (Maywood) 229: 988-995.

30. Pilsner JR, Hall MN, Liu X, Ahsan H, llievski V, et al. (2011) Associations of plasma selenium with arsenic and genomic methylation of leukocyte DNA in Bangladesh. Environ Health Perspect 119: 113-118.

31. Xiang N, Zhao R, Song G, Zhong W (2008) Selenite reactivates silenced genes by modifying DNA methylation and histones in prostate cancer cells. Carcinogenesis 29: 2175-2181

32. Fiala ES, Staretz ME, Pandya GA, El-Bayoumy K, Hamilton SR (1998) Inhibition of DNA cytosine methyltransferase by chemopreventive selenium compounds, determined by an improved assay for DNA cytosine methyltransferase and DNA cytosine methylation. Carcinogenesis 19: 597-604.

33. Bernard C (1974) Lectures on the phenomena common to animals and plants Trans Hoff HE, Guillemin R, Guillemin L, Springfield (IL): Charles C Thomas.

34. Gardner DK, Weissman A, Howles CM, Shoham Z (2012) Textbook of Assisted Reproductive Techniques. Volume One: Laboratory Perspectives. Fourth Edition, CRC Press.

35. Kralikova M, Melounova J, Crha I, Matejovicova M, Zakova J, et al. (2011) Intraindividual variability of homocysteine and related thiols concentrations in follicular fluid. J Assist Reprod Genet 28: 863-868.

Citation: Comhaire FH, Decleer WAE, Declerck K, Vanden Berghe W (2015) Investigation of Adverse Cancer Related DNA Methylation Variation in the Human Telomerase Reverse Transcriptase (hTERT) Promotor in Follicular Fluid Cells of Women Subjected to Ovarian Stimulation for In Vitro Fertilization (IVF). JFIV Reprod Med Genet 3: 161. doi:10.4172/2375-4508.1000161 